\title{
Is retardation of development of retina and brain a predictor of age-related macular degeneration and Alzheimer's disease?
}

\author{
N.A. Stefanova*, O.S. Kozhevnikova, D.V. Telegina, N.G. Kolosova \\ Institute of Cytology and Genetics SB RAS, Novosibirsk, Russia \\ *e-mail:stefanovan@bionet.nsc.ru
}

Key words: age-related macular degeneration, Alzheimer's disease, RNA sequencing, OXYS rats

Motivation and Aim: Alzheimer's disease (AD) and age-related macular degeneration (AMD) are two complex incurable neurodegenerative disorders the common pathogenesis of which is actively discussed. They manifest in the accumulation of amyloid beta $(\mathrm{A} \beta)$ in abnormal extracellular deposits: senile plaques in the brain of $\mathrm{AD}$ patients and drusen in the eyes of AMD patients. AD and AMD are complex diseases, formation of which is controlled by a variety of interacting genetic and environmental factors. Their manifestation occurs after the unfolding of the underlying events at the molecular level that are difficult to study in humans. Using senescence-accelerated OXYS rats, which simulate key characteristics of AMD and sporadic AD, we evaluated molecular and genetic background of the development of these diseases.

Methods and Algorithms: At preclinical (age 20 days), early (3-5 mo), and advanced (18 mo) stages of AMD- and AD-like pathologies in the retina and prefrontal cortex of OXYS and Wistar (control) rats, we evaluated differences in gene expression according to RNA sequencing (RNA-Seq) data to identify the metabolic processes and pathways, the changes in the activity of which lie at the basis of the transition from healthy aging to the development of AMD and AD.

Results: The manifestation of signs of AMD- and AD-like pathologies in OXYS rats (3-5 mo) is associated with change in the expression of genes associated with the immune system, inflammation, oxidative stress, calcium homeostasis, and apoptosis. Progression of the pathologies (3-18 mo) occurs against the background of alteration in expression of genes associated with the metabolic pathway of $\mathrm{AD}$, including those related to $\mathrm{A} \beta$ precursor protein processing, aggregation and degradation of $\mathrm{A} \beta$, synaptic processes, and mitochondrial dysfunction - in retina and cortex. Our results are consistent with RNA-Seq data on changes in the transcriptome of the prefrontal cortex of AD patients. Importantly, at the preclinical period in the retina and the cortex of OXYS rats, the expression of genes whose products are involved in the development of the central nervous system, synaptic transmission and neuronal plasticity is changed.

Conclusion: Our results suggest that retardation of the development of retina and brain may predict the emergence of the signs of AMD and AD in OXYS rats and, possibly, of these diseases in humans. This is supported by the data on the delayed hippocampal neurogenesis and reflex formation in early postnatal period as well as reduced duration of pregnancy ( $5 \%$ shorter than in Wistar, $p<0.0003$ ) in OXYS rats (Kozlova et al., 2018). Our results raise the question that preterm neonates may have a higher chance of $\mathrm{AMD}$ and $\mathrm{AD}$ development in the future even in the absence of cognitive impairments in early ontogenesis and retinopathy in prematurity.

Acknowledgements: This work was supported by a Russian Scientific Foundation grant (16-15-10005). 